\title{
Modelagem matemática aplicada à dinâmica de células imunológicas mediante a presença de populações tumorais
}

\author{
Louise Reips ${ }^{1}$ \\ Departamento de Matemática, UFSC, Blumenau, SC \\ Luiz Augusto Scheuermann França ${ }^{2}$ \\ Graduando em Engenharia de Controle e Automação, UFSC, Blumenau, SC \\ Rafael Aleixo ${ }^{3}$ \\ Departamento de Matemática, UFSC, Blumenau, SC
}

\begin{abstract}
Resumo. O câncer é uma patologia que tem como característica a mutação de células saudáveis em células anormais (tumorais), desenvolvendo-se de forma desordenada e agressiva. Na presente investigação, apresenta-se um modelo matemático que descreve a interação do sistema imunológico com as células tumorais. O modelo inclui a atuação e ativação de células da imunidade inata, como macrófagos dos tipos I e II, e da imunidade adaptativa, tais quais linfócitos $\mathrm{T}$ e B, bem como aborda a produção e secreção de citocinas pró e anti-inflamatórias por tais células. Para tal, fez-se uso de um modelo matemático proposto em [1] e novas equações referentes ao crescimento populacional celular de eosinófilos, basófilos e mastócitos, buscando enriquecer a representação do fenômeno biológico em questão.
\end{abstract}

Palavras-chave. Câncer, imunologia, modelagem matemática.

\section{Introdução}

O câncer é uma patologia com alta taxa de mortalidade global [8], não havendo causas específicas, surgindo da mutação de células saudáveis em células anormais (tumorais) através de um processo denominado oncogênese. A Agência Internacional de Pesquisa em Câncer (IARC) classifica, entre os agentes causadores, fatores internos (intrínsecos do genótipo do indivíduo) e fatores externos (relacionados ao meio) que podem colaborar para que tal proliferação de células venha a surgir no organismo. Estima-se ainda que uma parcela considerável de indivíduos acaba desenvolvendo a doença no processo de senilidade, visto que, na velhice, a reparação celular é menos eficaz. Estudos mostram que, mediante a implantação de estratégias de combate à doença em estágios iniciais da patologia, o tratamento se torna mais adequado, permitindo um potencial aumento na taxa de sobrevida do indivíduo, ressaltando a importância do estudo de tal fenômeno biológico.

Na busca por métodos alternativos à radioterapia e quimioterapia, pesquisas recentes de Institutos de Oncologia trazem consigo resultados promissores da aplicação de tratamentos imunoterápicos em indivíduos acometidos pelo câncer $[5,14]$. Nesse sentido, a imunoterapia é um trata-

\footnotetext{
${ }^{1}$ l.reips@ufsc.br

${ }^{2}$ scheuermannfranca@gmail.com

${ }^{3}$ rafael.aleixo@ufsc.br
} 
mento que fortalece o sistema imunológico do paciente forçando o próprio organismo a combater células tumorais sem a introdução de compostos químicos, não havendo efeitos colaterais negativos.

Com isso em vista, diversos modelos matemáticos buscam descrever esses processos através do uso de equações diferenciais $[2,7,11]$, na tentativa de aproximar corretamente a dinâmica da resposta imunológica inata-adaptativa frente a patógenos tumorais.

\section{O sistema imunológico}

Para compreender os efeitos da imunoterapia no organismo, é necessário, primeiramente, entender como o sistema imunológico funciona. O sistema imunológico é dividido em duas ramificações: a imunidade inata e a adaptativa. Os macrófagos, os neutrófilos e as células dendríticas (DCs) são exemplos de células que compõe o sistema inato imune, já os linfócitos tipos B e T fazem parte da imunidade adaptativa $[10,16]$. A composição desses dois sistemas tem como objetivo destruir de modo eficaz agentes infecciosos e substâncias nocivas ao corpo humano. Entre as incumbências da imunidade inata, pode-se citar a síntese de proteínas, citocinas e quiomicinas, além da fagocitose. Essa imunidade compõe um conjunto de tarefas inerentes ao ser humano, sem a introdução de compostos exteriores ao indivíduo. Já a resposta imune adaptativa atua no que se refere à ativação de células altamente especializadas, tendo como uma das principais características a memória imune, ou seja, tendo o indivíduo sido exposto a uma patologia infecciosa específica uma vez, futuramente, em caso de reincidência, seu organismo agirá de modo rápido e eficiente, identificando o agente causador e atuando contra ele.

O modelo matemático aqui proposto busca explorar a dinâmica de atuação do sistema imunológico, envolvendo citocinas (pró e anti-inflamatórias) atuantes na ativação ou inibição de células imunológicas [2], visto que possuem um papel fundamental no combate à proliferação de células tumorais $[10,19]$.

\section{Modelo matemático}

Conforme citado anteriormente, apresenta-se aqui um modelo matemático que inclui a atuação e ativação de células inatas, como macrófagos dos tipos I e II e células natural killers (NKs), bem como células imunes adaptativas (do tipo T-Helper e T-citotóxicas), além de englobar também a produção e secreção de citocinas pró-inflamatórias (IL-12, IFN- $\gamma$, TNF- $\alpha$ ) e anti-inflamatórias (IL-6, IL-10, IL-23, TGF- $\beta$ ) na atuação da resposta imunológica em populações de células tumorais. Para tal, faz-se uso de um modelo matemático proposto em [1] e são acrescentadas três novas equações, as quais referem-se à dinâmica de três células do sistema imune inato, sendo elas: eosinófilos, basófilos e mastócitos. A seguir, detalha-se cada uma das equações envolvidas, dando ênfase para as modificações sugeridas, com as respectivas considerações:

- $C_{i}$ representa o efeito de saturação de células cancerígenas e é dado por $C_{i}=\frac{C}{(k+C)}$. Esse termo está presente nas equações propostas, onde $k$ é o nível de saturação média de células cancerígenas estimuladas devido à resposta imune [7];

- O termo referente à proliferação das células, considera que as células tumorais e imunológicas têm crescimento logístico sob uma taxa $\alpha_{i}$, até uma capacidade de suporte $\beta_{i}$ [12];

- Células imunes adaptativas devem ser ativadas através da produção de compostos por outras células. As citocinas são substâncias que impulsionam a proliferação e ativação das células imunes [19], e podem ser produzidas por células imunes inatas, células T, neutrófilos, eosinófilos e basófilos [7];

- Todas as células que possuem alguma interação direta com as células tumorais necessitam de sua desativação, que ocorre a uma taxa $\delta_{i}$;

- As células modeladas têm um período de meia vida (semidesintegração) $\mu_{i}^{-1}$ e estão consideradas na parcela que representa a apoptose celular. 
- Equação para Macrófagos do tipo I $\left(M_{1}\right)$ e II $\left(M_{2}\right)$ :

Os macrófagos, de ambos os tipos, são ativados a uma taxa $\rho_{m}$ devido à interação com células tumorais [19] e à produção das interleucinas TNF- $\alpha$ e IL-10 por outras populações celulares. A segunda parcela em ambas as equações representa a proliferação de macrófagos do tipo I e II, que é inibida pelas interleucinas IL-23, TGF- $\beta$ e IFN- $\gamma$, a uma taxa $\eta_{3}$ e $\eta_{1}$ respectivamente. O fenômeno de apoptose também está presente nas equações abaixo, onde o parâmetro $\mu_{m}$ foi retirado de [4]. Diferentemente da equação que representa os macrófagos do tipo II (2), na equação (1) considera-se o termo $\delta_{m} M_{1} C$, com $\delta_{m}$ estimado em [6], que se refere à necrose, ou seja, a desativação devido à interação com as células tumorais pelo fato de macrófagos do tipo I produzirem citocinas próinflamatórias.

$$
\begin{aligned}
& \frac{d M_{1}}{d t}=\rho_{m} I_{\alpha} C_{i}+\frac{\alpha_{m} M_{1}\left(1-\frac{M_{1}}{\beta_{m}}\right)}{1+\eta_{3}\left(I_{\beta}+I_{23}\right)}-\delta_{m} M_{1} C-\mu_{m} M_{1}, \\
& \frac{d M_{2}}{d t}=\rho_{m} I_{10} C_{i}+\frac{\alpha_{m} M_{2}\left(1-\frac{M_{2}}{\beta_{m}}\right)}{1+\eta_{1} I_{\gamma}}-\mu_{m} M_{2} .
\end{aligned}
$$

\section{- Equação para células NK:}

As células NKs são ativadas a uma taxa $\rho_{k}$ devido à interação com células tumorais [19]. Novamente, a segunda parcela se refere à proliferação de células, sendo essa inibida pelas interleucinas IL-23, TGF- $\beta$, com parâmetros $\alpha_{K}$ retirado de [22] e $\beta_{k}$ de [12,20]. As parcelas seguintes se referem à necrose e apoptose, respectivamente, considerando uma taxa de desativação $\delta_{K}$ [6] e um período de meia vida $\mu_{k}[18]$.

$$
\frac{d N_{K}}{d t}=\rho_{K} I_{\alpha} C_{i}+\frac{\alpha_{K} N_{K}\left(1-\frac{N_{K}}{\beta_{K}}\right)}{1+\eta_{3}\left(I_{\beta}+I_{23}\right)}-\delta_{K} N_{K} C-\mu_{K} N_{K} .
$$

\section{- Equação para células T citotóxicas:}

As células $T$ citotóxicas são ativadas a uma taxa $\rho_{8}$ devido à interação com células tumorais [19] através da interleucina IL-12. A proliferação de células é inibida pela interleucina TGF- $\beta$ a uma taxa $\eta_{3}$ e os parâmetros $\alpha_{t}$ e $\beta_{t}$ foram retirados de [20,22]. A necrose está relacionada a uma taxa de desativação $\delta_{t}[6]$ e um período de meia vida $\mu_{8}[18]$.

$$
\frac{d T_{C}}{d t}=\rho_{8} I_{12} C_{i}+\frac{\alpha_{t} T_{C}\left(1-\frac{T_{C}}{\beta_{t}}\right)}{1+\eta_{3} I_{\beta}}-\delta_{t} T_{C} C-\mu_{8} T_{C} .
$$

\section{- Equação para células T Helper Th0, Th1, Th2 e Th17:}

As células T Helper Th0 são ativadas a uma taxa $\rho_{t}$ devido à interação com células tumorais [19] pela interleucina IL-12. Essas células (Th0) possuem uma particularidade, pois se diferenciam em células Th1, Th2 e Th17, que serão modeladas implicitamente como segue, e são ativadas à mesma taxa mas por interleucinas diferentes. A proliferação dessas células é inibida por interleucinas diferentes e a desativação está relacionada a uma taxa $\delta_{t}[6]$ e um período de meia vida $\mu_{t}[18]$. As 
equações referentes a essas células são dadas abaixo:

$$
\begin{aligned}
\frac{d T_{0}}{d t}= & \rho_{t} I_{12} C_{i}+\frac{\alpha_{t} T_{0}\left(1-\frac{T_{0}}{\beta_{t}}\right)}{1+\eta_{3} I_{\beta}}-\delta_{t} T_{0} C-\mu_{t} T_{0}, \\
\frac{d T_{1}}{d t}= & \rho_{t} I_{12} T_{0}+\frac{\alpha_{t} T_{1}\left(1-\frac{T_{1}}{\beta_{t}}\right)}{1+\left(\eta_{3} I_{\beta}+\eta_{2} I_{4}\right)}-\delta_{t} T_{1} C-\mu_{t} T_{1}, \\
\frac{d T_{2}}{d t}= & \rho_{t} I_{4} T_{0}+\frac{\alpha_{t} T_{2}\left(1-\frac{T_{2}}{\beta_{t}}\right)}{1+\eta_{3} I_{\beta}}-\mu_{t} T_{2}, \\
\frac{d T_{17}}{d t}= & \rho_{t} I_{6} T_{0}+\frac{\alpha_{t} T_{17}\left(1-\frac{T_{17}}{\beta_{t}}\right)}{1+\eta_{1} I_{\gamma}}-\mu_{t} T_{17} .
\end{aligned}
$$

\subsection{Equação para eosinófilos}

Os eosinófilos, bem como basófilos e mastócitos, podem produzir quantidades abundantes de IL-4, uma citocina que é sintetizada pelas células Th2. Além disso, eosinófilos também produzem citocinas do tipo IL-15, que são importantes no processo de crescimento tumoral visto que atuam no fator de crescimento de células NKs, proliferando-as e aumentando a liberação de interferon- $\gamma$ (IFN- $\gamma$ ) pelas NKs. O IFN- $\gamma$ é uma citocina capaz de ativar os macrófagos e é eficaz na inibição e progressão de tumores.

O fator de necrose tumoral (TNF) atua diretamente na apoptose dos eosinófilos [17], porém, não há pesquisas consistentes que demonstram como de fato isso ocorre. Tumores sólidos ou hematológicos (benignos ou malignos) são apontados como uma das causas comuns de eosinofilia, logo, fica evidente a importância de acrescentar-se tal equação no estudo de crescimento tumoral. Dessa forma, apesar dos eosinófilos atuarem diretamente em infecções parasitárias e reações alérgicas, indiretamente são ativados pelas células Th2 e influenciam as células NKs e macrófagos na presença de células tumorais [21]. Dessa forma, a equação referente aos eosinófilos, ignorando a ativação devido à processos parasitários, é dada por:

$$
\frac{d E}{d t}=\phi_{E} I_{5} C_{i}+\frac{\alpha_{E} E\left(1-\frac{E}{\beta_{E}}\right)}{1+\eta_{3} I_{\beta}}-\delta_{E} E C-\mu_{E} E .
$$

Os eosinófilos são ativados a uma taxa $\phi_{E}$ devido à presença de células cancerígenas [19] e ativados por células $\mathrm{T}$ através da produção de citocinas do tipo IL-5. A segunda parcela se refere à proliferação da população eosinofílica. Os eosinófilos crescem a uma taxa $\alpha_{E}$ até uma capacidade $\beta_{E}$, estimulada por IFN- $\gamma$, secretada pelas células NKs [19]. A proliferação dos eosinófilos é inibida a uma taxa $\eta_{3}$, devido à citocinas TGF- $\beta$ [15]. Os eosinófilos são desativados devido à interação com células cancerígenas a uma taxa $\delta_{E}[6]$ e tem uma meia vida de $\mu_{E}^{-1}[9]$, que correspondem as parcelas de necrose e apoptose (via receptores de morte), respectivamente.

\subsection{Equação para mastócitos}

Os mastócitos são células produtoras da interleucina IL-4, a qual interfere diretamente em células Th2, podendo atuar de modo inibidor ou promotor de células tumorais, dependendo do tipo patológico em questão [10], especialmente porque detém a capacidade de produzir substâncias que favorecem o desenvolvimento da angiogênese. Para esse estudo, considerou-se apenas a contribuição positiva dessas células, ignorando as contribuições relacionadas ao favorecimento da progressão tumoral. Certas doenças, como problemas cardiovasculares, cânceres e infecções agudas, acarretam 
a liberação de intercessores mastocitários que atuam na resposta imunológica do paciente e, por esse motivo, inclui-se aqui uma equação para representar a contribuição de tais células no processo do estudo do desenvolvimento tumoral.

Os mastócitos originam-se na medula óssea através de um célula precursora ainda desconhecida [10,16] e produzem as interleucinas IL-5 (cujas células receptoras são os eosinófilos e linfócitos B), IL-10 (que tem como função a ativação de linfócitos B, mastócitos e Th2), além de IL-13. A equação referente aos mastócitos é dada como:

$$
\frac{d M_{A}}{d t}=\phi_{M_{A}} I_{\alpha} C_{i}+\frac{\alpha_{M_{A}} M_{A}\left(1-\frac{M_{A}}{\beta_{M_{A}}}\right)}{1+\eta_{3} I_{\beta}}-\delta_{M_{A}} M_{A} C-\mu_{M_{A}} M_{A} .
$$

Os mastócitos são ativados a uma taxa $\phi_{M_{A}}[13,19]$ devido à presença de células cancerígenas, consideradas na primeira parcela da equação. O modelo considera, ainda, que a população mastocitária cresce a uma taxa $\alpha_{M_{A}}[22]$ até uma capacidade $\beta_{M_{A}}$ [20] e sua proliferação é inibida a uma taxa $\eta_{3}$, devido às citocinas TGF- $\beta$ [10]. Os mastócitos são desativados a uma taxa $\delta_{M_{A}}[6]$ e tem uma meia-vida de $\mu_{M_{A}}^{-1}[9]$.

\subsection{Equação para basófilos}

Os basófilos correspondem de 0 a $2 \%$ do número total de leucócitos circulantes e estão associados a respostas do sistema imunológico contra parasitas (juntamente com os eosinófilos) e alérgenos. Porém, como respostas a alguns tipos específicos de doenças como o câncer, podem ocasionar um aumento considerável do número de basófilos no sangue (basofilia), devido à presença de processos inflamatórios [10]. Eles são responsáveis pela produção de interleucinas IL-4, que possuem a função de diferenciar células Th0 em Th2.

Como a presente investigação ponderou apenas processos tumorais, foi ignorada a ativação dessas células devido à processos alérgenos, levando em consideração apenas a influência de células NKs na população de basófilos. Sendo assim, a equação para os basófilos é dada por:

$$
\frac{d B}{d t}=\phi_{B} I_{8} C_{i}+\frac{\alpha_{B} B\left(1-\frac{B}{\beta_{B}}\right)}{1+\eta_{3} I_{\beta}}-\delta_{B} B C-\mu_{B} B
$$

Os basófilos são ativados a uma taxa $\phi_{B}[13,19]$ devido à presença de células cancerígenas e crescem a uma taxa $\alpha_{B}$ [22] até uma capacidade $\beta_{B}$ [20], com sua proliferação inibida a uma taxa $\eta_{3}$, devido à citocinas TGF- $\beta$ [10]. Os mastócitos são desativados a uma taxa $\delta_{B}[6]$ e tem uma meia-vida de $\mu_{B}^{-1}[9]$.

\subsection{Equação para células cancerígenas}

As células cancerígenas crescem a uma taxa $\alpha_{C}$, até uma taxa de capacidade $\beta_{C}[4,12,20]$. A segunda parcela se refere à atuação de macrófagos $M_{2}$, que impulsionam o crescimento da população tumoral a uma taxa $\theta_{C}$ [4]. Outras células cancerígenas podem ser fagocitadas por macrófagos do tipo 1 e destruídas pelas células efetoras como células citotóxicas, NKs, Th1, eosinófilos, basófilos e mastócitos, a uma taxa $\Lambda_{C}$ [4], podendo inibir essa desativação através da produção de citocinas TGF- $\beta$ [13]. Além disso, considera-se aqui o termo de apoptose a uma taxa $\mu_{C}$ estimada em [3]. A equação referente as células cancerígenas é fornecida por:

$$
\frac{d C}{d t}=\alpha_{C} C\left(1-\frac{C}{\beta_{C}}\right)+\theta_{C} M_{2} C+\theta_{1} P_{C}-\left[\frac{\Lambda_{C}\left(M_{1}+N_{K}+T_{C}+T_{1}+E+B+M_{A}\right)}{1+\eta_{3} I_{B}}+\mu_{C}\right] C
$$




\section{Resultados numéricos}

Para o sistema de equações diferenciais aqui proposto, considerou-se como condições iniciais o fato de que a população de células cancerígenas e células pertencentes ao sistema inato são diferentes de zero; já a população inicial de células referentes ao sistema imune adaptativo é considerada nula. Assumiu-se ainda que todas as soluções são não negativas para $t \geq 0$. Todos os parâmetros utilizados foram retirados da literatura, conforme experimentos relatados e podem ser facilmente encontrados nas referências citadas anteriormente. Mostra-se, abaixo, o crescimento populacional de células tumorais, além de eosinófilos, basófilos e mastócitos, fazendo uso do método numérico de Runge-Kutta de quarta ordem.

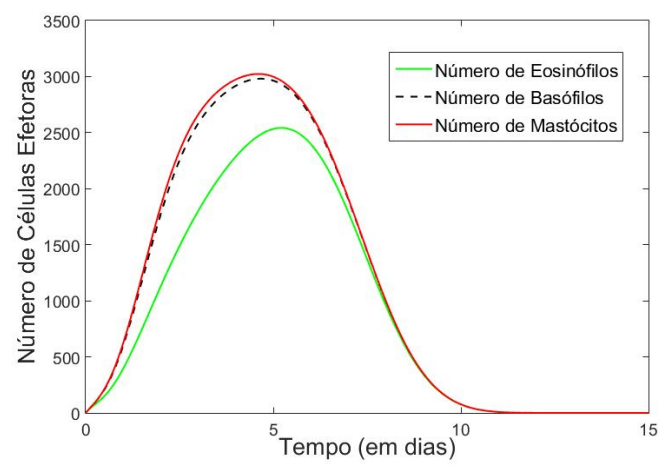

(a) População de eosinófilos, basófilos e mastócitos

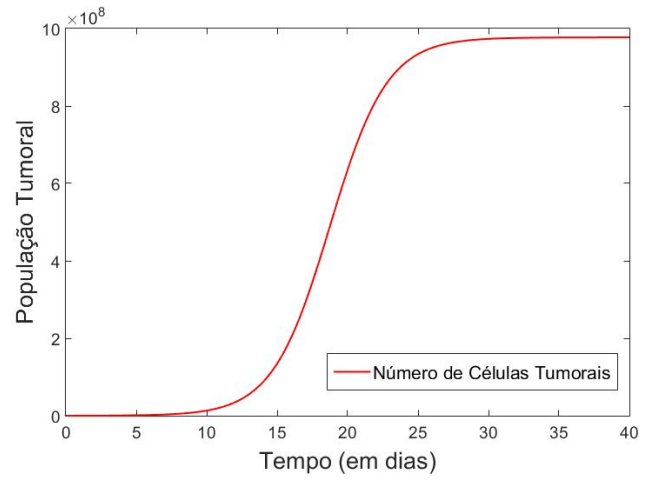

(b) População de células tumorais

Figura 1: Crescimento populacional de células tumorais, eosinófilos, basófilos e mastócitos.

\section{Conclusões}

Apresentamos um modelo matemático que descreve a dinâmica da resposta imunológica inataadaptativa frente a patógenos tumorais. O modelo proposto, quando comparado a outros encontrados na literatura atualmente, apresenta novas equações referentes ao crescimento populacional celular de eosinófilos, basófilos e mastócitos. Tais equações levam em consideração uma extensa pesquisa bibliográfica para incluir as respectivas interleucinas concernentes relatadas em artigos médicos e biológicos encontrados na literatura. Experimentos numéricos apresentam os gráficos de crescimento populacional para essas três populações celulares e sua atuação na presença de células cancerígenas, com resultados que corroboram aos apresentados em [4] relativos à população celular tumoral.

\section{Referências}

[1] Amima, I. M. Investigating tumour micro environment dynamics based on cytokine-mediated innate-adaptive immunity, Dissertação de Mestrado, Stellenbosch University, 2018.

[2] Bellomo, N. and Preziosi, L. Modelling and mathematical problems related to tumor evolution and its interaction with the immune system, Mathematical and Computer Modelling, 32:413452, 2000. DOI: 10.1016/S0895-7177(00)00143-6.

[3] Benzekry, S., Lamont, C. et al. Classical mathematical models for description and prediction of experimental tumor growth, PLoS Computational Biology, 10:e1003800, 2014. DOI: 10.1371/journal.pcbi.1003800. 
[4] Breems, N. Y. den and Eftimie, R. The re-polarisation of M2 and M1 macrophages and its role on cancer outcomes, Journal of Theoretical Biology, 390:23-39, 2016. DOI: 10.1016/j.jtbi.2015.10.034.

[5] Byrne, A. , Savas, P., et al. Tissue-resident memory T cells in breast cancer control and immunotherapy responses, Nature R. Clinical Oncology, 2020. DOI: 10.1038/s41571-020-0333y.

[6] Diefenbach, A. , Jensen, E. R., Jamieson, A. M. and Raulet, D. H. Rae1 and H60 ligands of the NKG2d receptor stimulate tumour immunity, Nature, 413:165-171, 2001. DOI: $10.1038 / 35093109$.

[7] Eftimie, R. and Hamam, H. Modelling and investigation of the CD4+ T cells-macrophages paradox in melanoma immunotherapies, Journal of Theoretical Biology, 420:82-104, 2017. DOI: $10.1016 /$ j.jtbi.2017.02.022.

[8] Gupta, S., Harper, A., Ruan, Y., Barr, R., Frazier, A. L., Ferlay, J, Steliarova-Foucher, E. and Fidler-Benaoudia, M .M. International trends in the incidence of cancer among adolescents and young adults, J. of the National Cancer Institute, 2020. DOI: 10.1093/jnci/djaa007.

[9] Keohane, E. M., Rodak, B. F., Smith, L. J. and Walenga, J. M. Rodak's Hematology: clinical principles and applications, 5a. edição. Elsevier Saunders, 2016.

[10] Kindt, T. J., Goldsby, R. A., et al. Kuby Immunology, 6a. edição. W. H. Freeman, 2007.

[11] Kirschner, D. and Panetta, J. C. Modeling immunotherapy of the tumor-immune interaction, Journal of Mathematical Biology, 37:235-252, 1998. DOI: 10.1007/s002850050127.

[12] Kuznetsov, V. A., Makalkin, I. A., Taylor, M. A and Perelson, A. S. Nonlinear dynamics of immunogenic tumors: parameter estimation and global bifurcation analysis, Bulletin of Mathematical Biology, 56: 295-321, 1994. DOI: 10.1016/S0092-8240(05)80260-5.

[13] Lippitz, B. E. Cytokine patterns in patients with cancer: a systematic review, The Lancet Oncology, 14:e218-e228, 2013. DOI: 10.1016/S1470-2045(12)70582-X.

[14] Liu, E., Marin, D., et al. Use of CAR-Transduced Natural Killer Cells in CD19-Positive Lymphoid Tumors, The New England Journal of Medicine, 382:545-553, 2020. DOI: 10.1056/NEJMoa1910607.

[15] Mumm, J. and Oft, M. Cytokine-based transformation of immune surveillance into tumorpromoting inflammation, Oncogene, 27:5913-5919, 2008. DOI: 10.1038/onc.2008.275.

[16] Murphy, K. Imunobiologia de Janeway, 8a. edição. Tradução: D. C. Machado, G. Renard, L. P. Gualdi; revisão técnica: D. C. Machado. Artmed, Porto Alegre, 2014.

[17] Park, Y. M. and Bochner, B. S. Eosinophil Survival and Apoptosis in Health and Disease, Allergy Asthma Immunology Research, 2:87-101, 2010. DOI: 10.4168/aair.2010.2.2.87.

[18] Pillis, L. G., Radunskaya,A. E. and Wiseman, C. L. A validated mathematical model of cell-mediated immune response to tumor growth, Cancer Research, 65:7950-7958, 2005. DOI: 10.1158/0008-5472.CAN-05-0564.

[19] Sompayrac, L. M. How the immune system works. John Wiley \& Sons, Iowa, 2015.

[20] Uhr, J. W., Tucker, T., et al. Cancer dormancy: studies of the murine BCL1 lymphoma, Cancer Research, 51:5045s-5053s, 1991.

[21] Weller, P. F. and Lim, K. Human eosinophil-lymphocyte interactions, Mem. Inst. Oswaldo Cruz, 92:173-182, 1997. DOI: 10.1590/S0074-02761997000800023.

[22] Wilkie, K. P. and Hahnfeldt, P. Tumor-immune dynamics regulated in the microenvironment inform the transient nature of immune-induced tumor dormancy, Cancer Research, 73:35343544, 2013. DOI: 10.1158/0008-5472.CAN-12-4590. 